\title{
Generation of a long acting GCSF for treatment of neutropenia and stem cell harvest
}

\author{
Abdulrahman Alshehri, Richard Ross and lan R Wilkinson \\ Department of Human Metabolism, School of Medicine, Royal Hallamshire Hospital, \\ University of Sheffield, UK.
}

\section{Background}

Over the last 20 years, granulocyte colony-stimulating factor (GCSF) has become a recognized therapy in the treatment of patients suffering from neutropenia. Current therapies require daily injections of GCSF to stimulate stem cell production and response to treatment is often unpredictable as GCSF is rapidly cleared. A number of approaches to reducing GCSF clearance have been tried mainly through conjugation with another moiety. The technologies already being employed, include PEGylation, linking to immunoglobulins and glycosylation to increase the half-life of rhGCSF. However although these approaches have reduced clearance the pharmacokinetic profile has remained unpredictable.

\section{Aim and hypothesis}

The aim of this project is to create a long acting GCSF with predictable pharmacokinetic profile to provide a more effective treatment for generating HSCs for bone marrow transplantation. We will achieve this via incorporation of variable glycosylated linkers between two GCSF molecules. This will create a hyperglycosylated construct with a high molecular weight and protected from proteolysis resulting in reduced clearance while retaining bioactivity. This approach also alleviates potential problems with direct glycosylation of the ligand which may inhibit bioactivity and potentially introduce immunogenic sites.

\section{Methodology}

GCSF tandem molecules with linkers containing between 2-8 NAT glycosylation motifs and their respective controls ( $Q$ replaces $N$ in the sequence motif NAT) were cloned, and sequenced (Figure 1 \& Table 1). Following expression in Chinese hamster ovary (CHO) cells, expressed protein was quantified by ELISA and analysed by western blot and SDS-PAGE to confirm molecular weights. Bioactivity of purified proteins was tested using AML-193 proliferation assay.

\section{Figure 1:}

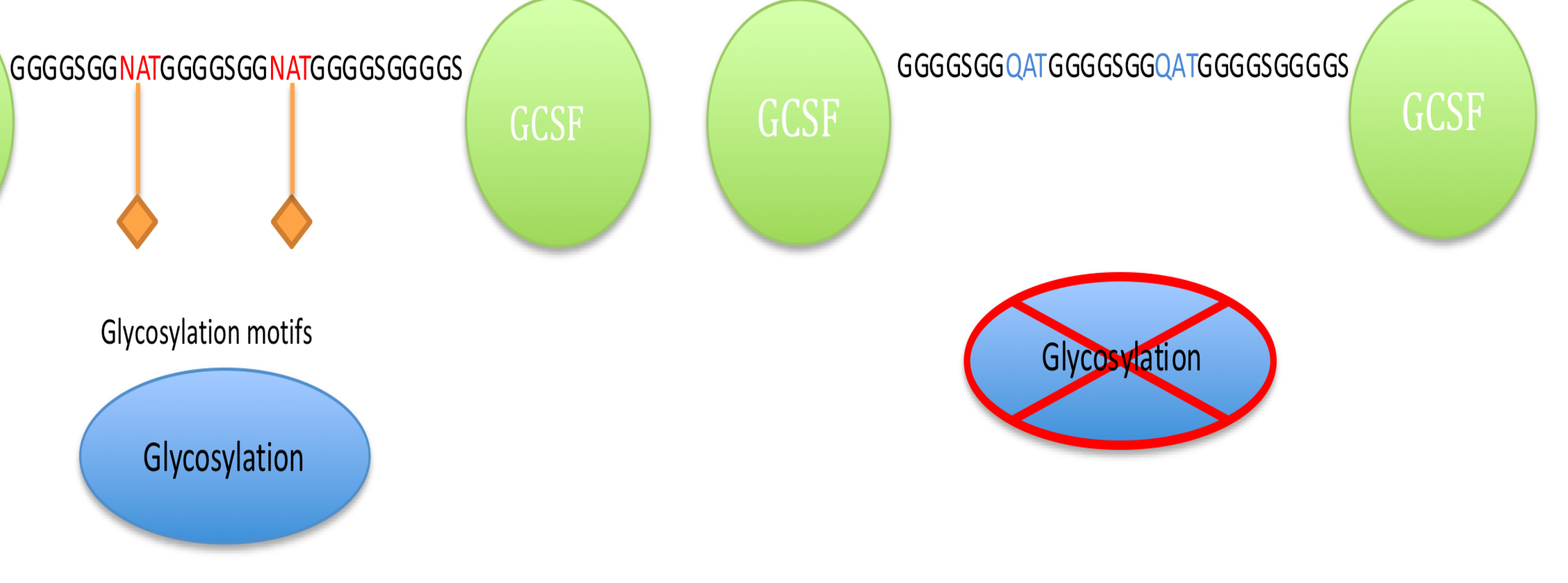

Table 1: List of constructs

\begin{tabular}{|l|c|c|c|}
\hline $\begin{array}{c}\text { Construct } \\
\text { name }\end{array}$ & $\begin{array}{c}\text { Number of NAT/ } \\
\text { QAT motifs in } \\
\text { linker region }\end{array}$ & $\begin{array}{c}\text { Molecular } \\
\text { weight } \\
(\mathrm{kDa})\end{array}$ & \multicolumn{1}{|c|}{ Description } \\
\hline GCSF (2NAT) & $2 \times$ NAT motifs & $\mathbf{4 5 . 2}$ & GCSF tandem agonist \\
\hline GCSF (2QAT) & $2 \times$ QAT motifs & $\mathbf{4 5 . 4}$ & Control for GCSF (2NAT) \\
\hline GCSF (4NAT) & $4 \times$ NAT motifs & 45.2 & GCSF tandem agonist \\
\hline GCSF (4QAT) & $4 \times$ QAT motifs & 45.4 & Control for GCSF (4NAT) \\
\hline GCSF (8NAT) & $8 \times$ NAT motifs & 48.5 & GCSF tandem agonist \\
\hline GCSF (8QAT) & $8 \times$ QAT motifs & 48.5 & Control for GCSF (8NAT) \\
\hline
\end{tabular}

\section{Results}

\section{Figure 2: Western blot \& SDS-PAGE analysis}

It is possible to express GCSF tandem molecules linked by a flexible linker $\left(\mathrm{Gly}_{4} \mathrm{Ser}\right)_{n}$ in mammalian cell lines. The glycosylated tandem molecules show an increased in molecular weight above that of their controls (non-glycosylated tandem molecules).

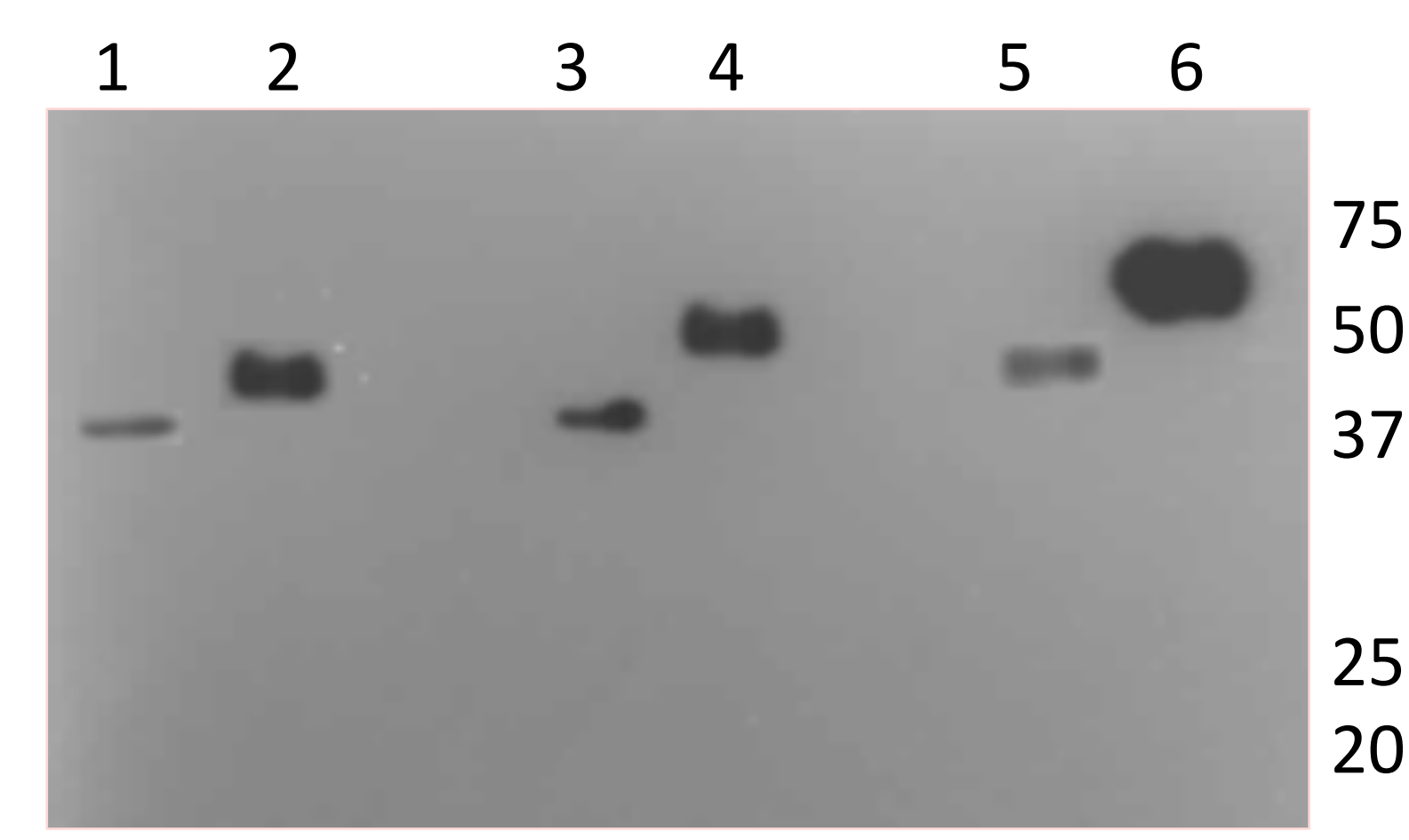

Western blot of crude media from transiently transfected $\mathrm{CHO}$ Flp-In cells expressing GCSF tandem molecules. Lane 1; GCSF (2QAT). Lane 2; GCSF (2NAT). Lane 3; GCSF (4QAT). Lane 4; GCSF (4NAT). Lane 5; GCSF (8QAT). Lane 6; GCSF (8NAT).

\section{Figure 3: AML-193 proliferation assay}

GCSF stimulates the proliferation of the AML-193 cell line (Human acute myeloid leukemic cell line). All purified GCSF tandems show increased bioactivity with all standard curves shifted to the left in comparison to native GCSF.

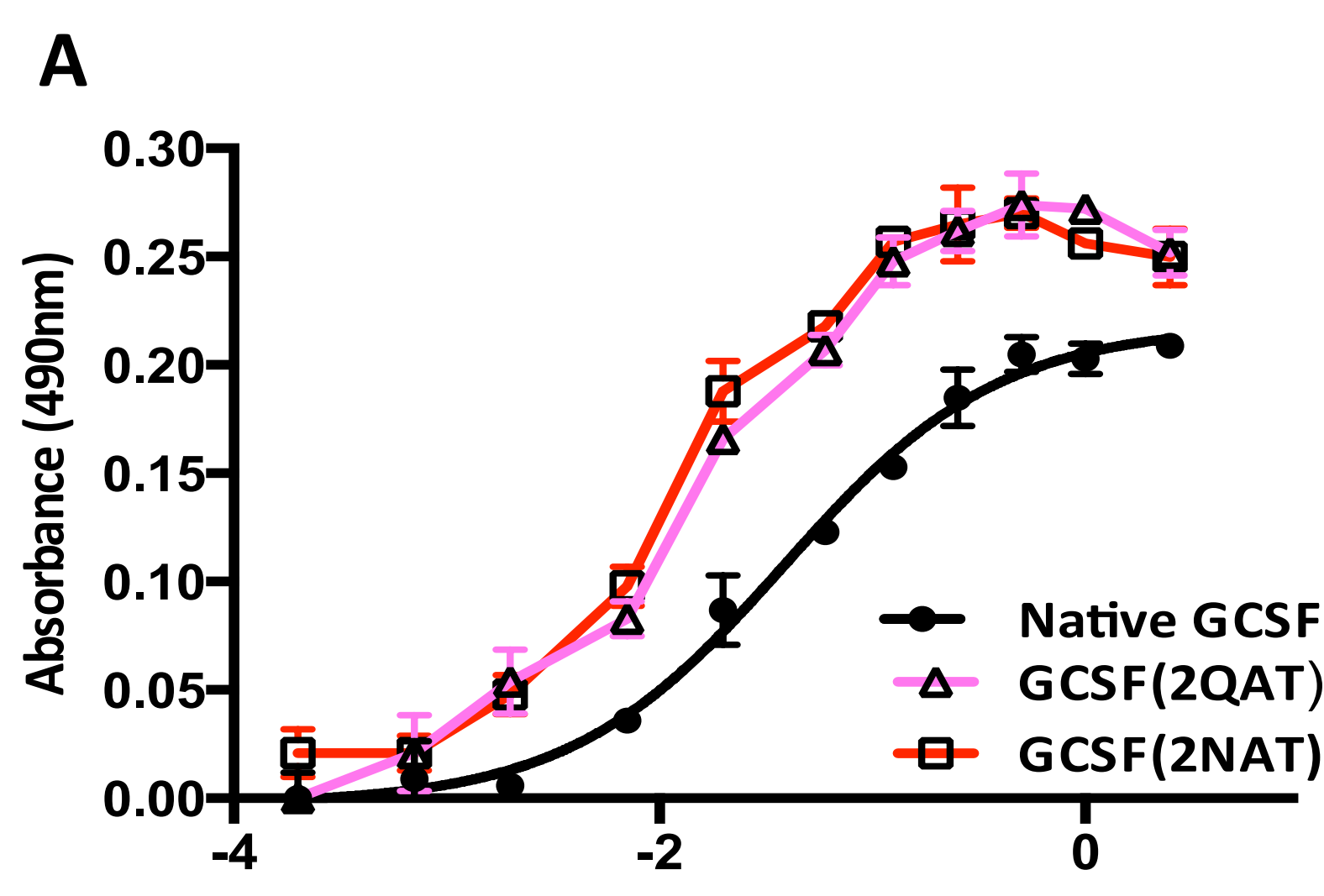

$\log (\mathrm{nM})$

$$
\text { C }
$$

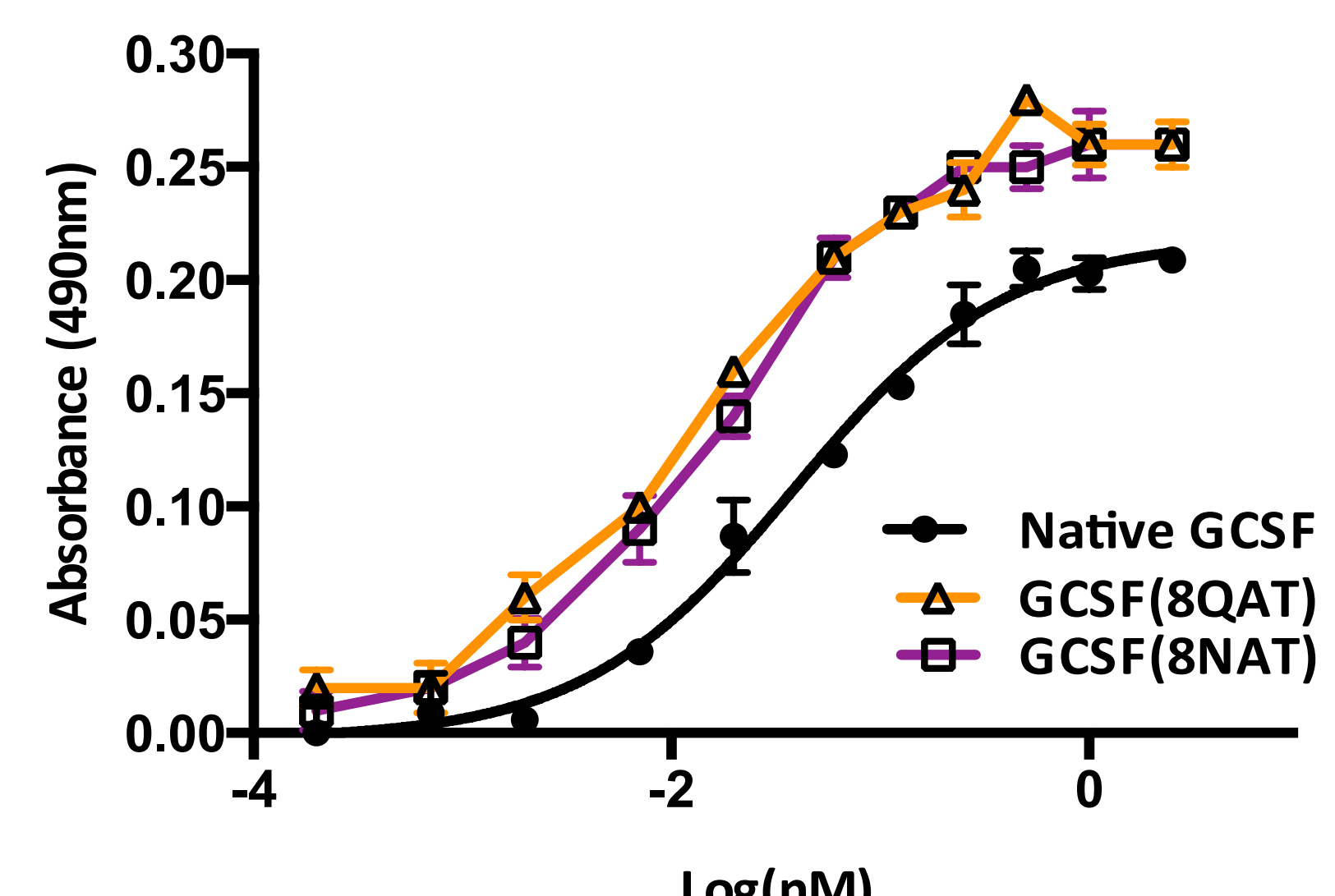

Figure 3 (A,B,C): Proliferation of AML-193 cells in the presence of GCSF tandems and native GCSF.

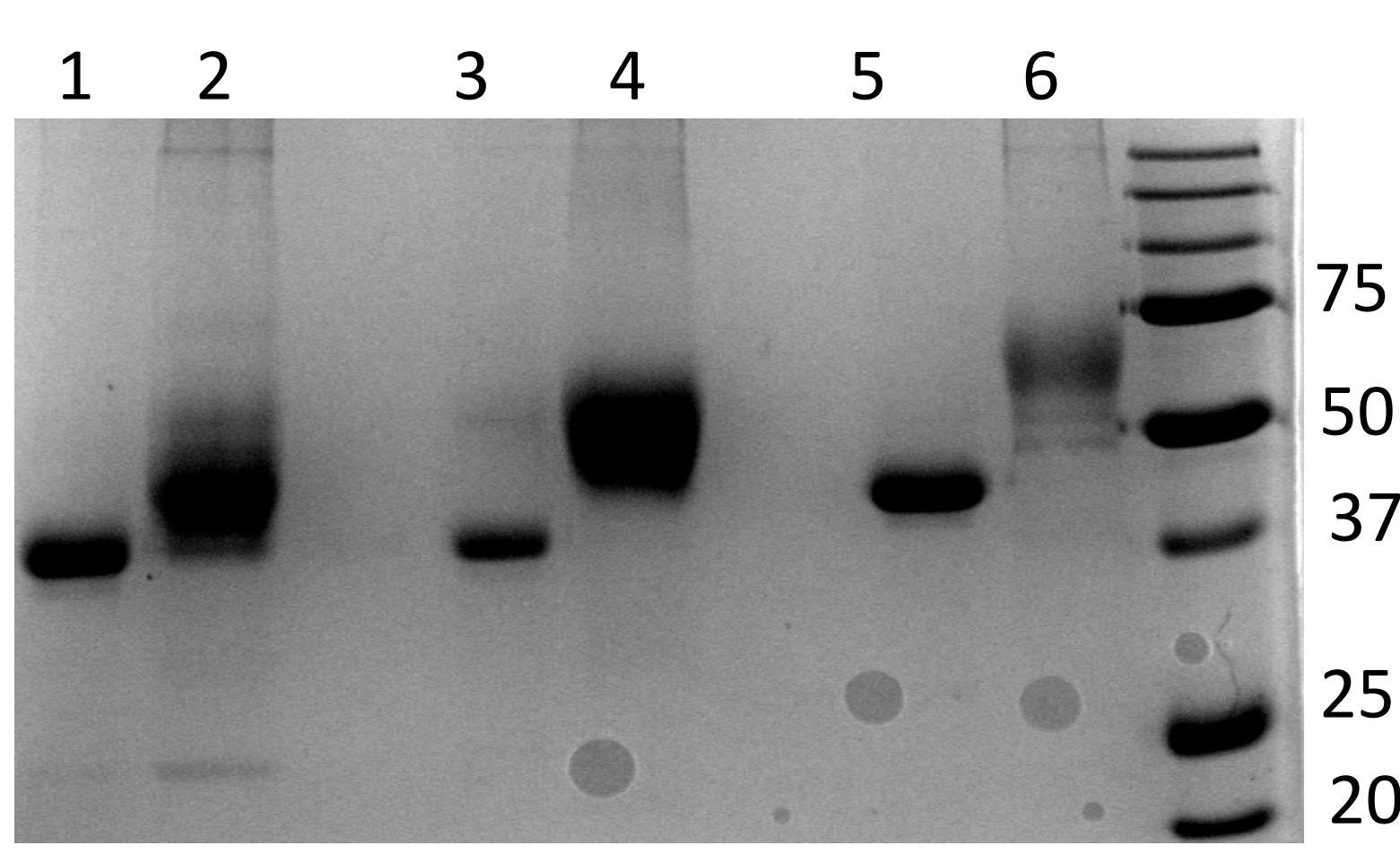

Purified GCSF tandem molecules analyzed by SDS-PAGE. Lane 1; GCSF (2QAT). Lane 2; GCSF (2NAT). Lane 3; GCSF (4QAT). Lane 4; GCSF (4NAT). Lane 5; GCSF (8QAT). Lane 6; GCSF (8NAT).

\section{Conclusion}

Results show that the use of glycosylated linkers to generate GCSF tandems results in molecules with increased molecular weight and increased bioactivity compared to native GCSF. Future studies will test protein clearance using a rat model system.

\section{References}

1. Yin et al. Bcl-xL is a dominant antiapoptotic protein that inhibits homoharringtonine-induced apoptosis in leukaemia cells. Mol. Pharmacol. 2011; 79, 1072-83

2. Li H, d'Anjou M. Pharmacological significance of glycosylation in therapeutic proteins. Current Opinion in Biotechnology. 2009; 20(6):678-84.

3. Sinclair AM, Elliott S. Glycoengineering: The effect of glycosylation on the properties of therapeutic proteins. Journal of Pharmaceutical Sciences. [Review]. 2005 Aug; 94(8):1626-35. 